\title{
Axisymmetric Thermo-elastic Deformation of the Cylinder with Two-dimensional Inhomogeneity of Material
}

\author{
V.I. Andreev \\ Moscow State University of Civil Engineering, Yaroslavskoye sh., 26, 129337 Moscow, Russia \\ asv@mgsu.ru
}

\begin{abstract}
The article discusses the use of a numerical method the calculation of finite cylinders into account the dependence of physical and mechanical properties of the material on temperature. If we have two-dimensional temperature field characteristics of the material depends on two coordinates. $-r$ and $z$ from which follows that the problem of thermoelasticity is also a two-dimensional. Using the numerical method allows to solve the problem for any state of the cylinder (plane stress or plane strain) and consider arbitrary boundary conditions at its ends.
\end{abstract}

Keywords: cylindrical shell, reinforced concrete, heat conductivity, thermoelasticity, inhomogeneity.

\section{Introduction}

In [1-6] considered the problem of calculating thick-walled cylinders under the action of force and temperature loads at one-dimensional (radial) inhomogeneity of the material. Temperature inhomogeneity was due to axisymmetric not varying along the axis of the cylinder temperature field. In many practical problems, a heat source inside the cylinder can be regarded as a point. In this case, the temperature field remaining axisymmetric varies along the cylinder axis, resulting in a two dimensional an inhomogeneity due to which you cannot apply a numerical-analytical method $[1,7]$.

\section{State of the Problem}

As an example, below we consider the calculation of stresses in the radiation-heat shield of the reactor plant. This design, also known as "dry protection," designed to reduce the effects of radiation and heat generated when operation of the reactor. In constructional sense thermal protection is fixed at the base a thick-walled cylindrical shell made of heat-resistant reinforced concrete [8]. Fig. 1 shows the design scheme of the problem. In constructional sense thermal protection is a fixed at the base thick-walled cylindrical shell made of heat-resistant reinforced concrete. Fig. 1 shows the design scheme of the problem.

To reduce the thermal load there is envisage prorolling air cooling in the clearances between the shell of the reactor, the design of thermal protection and biological protection of the reactor. To solve the problem is used the variational-difference method [9-11], while this method is used for solving the problem of heat conduction subject to the availability of internal sources of heat and coefficient of thermal conductivity depending on the temperature, and to solve the problem of determining the stress-strain state of the cylinder.

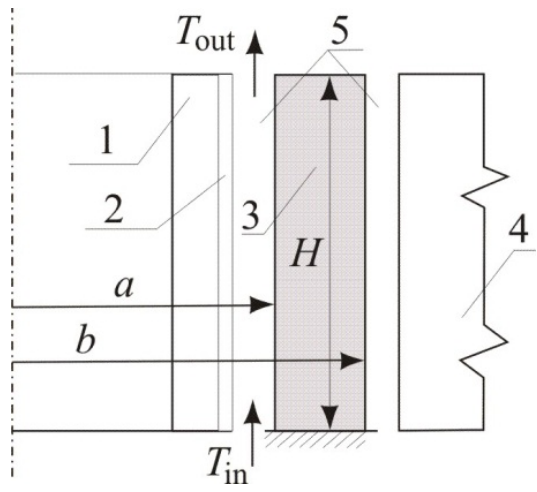

Fig. 1. Design scheme of heat shield: 1 - reactor vessel; 2 - heat insulation;

3 - radiation-heat shield; 4 - biological shield;

5 - cooling channels 


\section{Solution of the Problem of Heat Conduction}

In the stationary temperature field heat equation

$$
\lambda \nabla^{2} T+W(r, z)=0
$$

for the axisymmetric problem in cylindrical coordinates, taking into account the dependence $F(h)$ is written as

$$
\frac{1}{r} \frac{\partial}{\partial r}\left[r \lambda_{T}(T) \frac{\partial T}{\partial r}\right]+\frac{\partial}{\partial z}\left[\lambda_{T}(T) \frac{\partial T}{\partial z}\right]=-W(r, z)
$$

where $\lambda_{T}$ - coefficient of thermal conductivity, $W$ - the intensity of heat sources per unit volume.

For the function $W(r, z)$, describing the density of the internal heat sources caused by ionizing radiation, use dependence [12]:

$$
W(r, z)=W_{0}+W_{1} \exp [-\delta(r-a)] \sin \frac{\pi z}{H}
$$

in which $W_{0}, W_{1}$ and $\delta$ - empirical parameters.

The boundary conditions on the side and end surfaces of the cylinder taken as:

$$
\begin{gathered}
r=a ; \quad-\lambda_{T} \frac{\partial T}{\partial r}=\alpha_{a}\left(T-T_{c p}^{a}\right) ; \\
r=b ; \quad-\lambda_{T} \frac{\partial T}{\partial r}=\alpha_{b}\left(T-T_{c p}^{b}\right) ; \\
z=H ; \quad-\lambda_{T} \frac{\partial T}{\partial z}=\alpha_{H}\left(T-T_{c p}^{H}\right) ; \\
z=0 ; \quad T=T_{0}(r) .
\end{gathered}
$$

Here $T_{a m b}^{a}, T_{a m b}^{b}, T_{a m b}^{H}-$ ambient temperature (cooling air) onto the side surfaces and upper (end) surface; $\alpha_{a}, \alpha_{b}, \alpha_{H}$ - heat transfer coefficients for these surfaces are listed in [13]. The last boundary condition of the first kind can be accepted, given that the mass base is significantly greater than the mass the structure under consideration, and the temperature at the contact of two bodies - a known function $T_{0}(r)$.

Dependence $\lambda_{T}(T)$ approximated by a polynomial of the type

$$
\lambda_{T}(T)=\lambda_{0} \cdot \sum_{n=0}^{N} \beta_{n} T^{n}
$$

on the basis of the data presented in [14].

Fig. 2 shows the graphs of the internal heat source constructed in accordance with (3). Fig.3 shows temperature dependences taken into account that the temperature of the cooling air in channel height varies linearly, at values $\lambda_{T 0}=1.5 \mathrm{~W} /\left(\mathrm{m} \cdot{ }^{\mathrm{o}} \mathrm{C}\right)$;
$T_{\text {in }}=30{ }^{\circ} \mathrm{C}$; and $T_{\text {out }}=80^{\circ} \mathrm{C}$. The dimensions of the cylinder are: $a=2 \mathrm{~m}, b=H=3 \mathrm{~m}$.

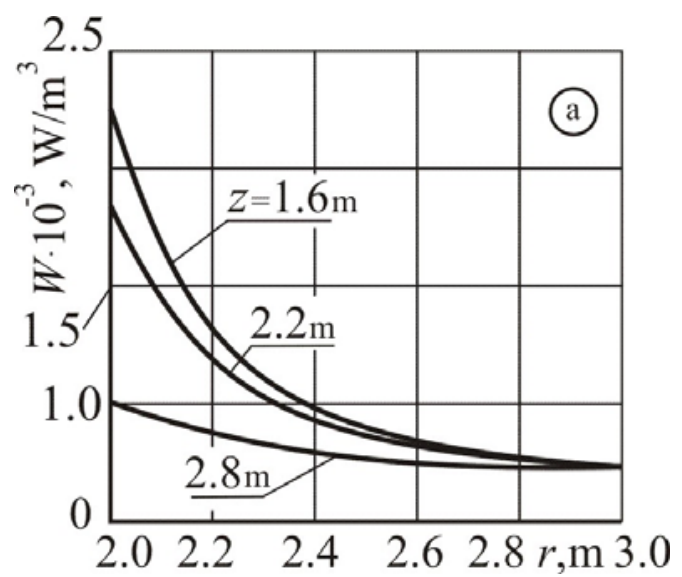

Fig. 2. Graph of the internal heat sources at different levels of the cylinder:

On the basis of performed calculations it can be concluded that in this temperature range the dependence $\lambda_{T}=\lambda_{T}(T)$ leads to a slight increase of temperature (not more $10 \%$ ).

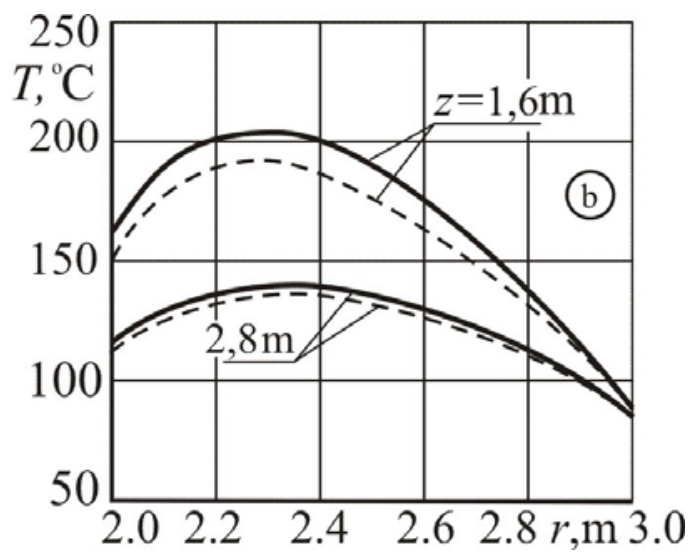

Fig. 3. Temperature dependences at different levels of the cylinder.

- - - Homogeneous material $-\lambda_{T}=\lambda_{T 0}$;

- Inhomogeneous material $-\lambda_{T}=\lambda_{T}(T)$

The solution of the temperature problem by a variation-difference method is convenient from the point of view that the resulting calculation of net function $T\left(r_{i}, z_{j}\right)$ and its gradient is used in the future to solve the problem of thermoelasticity in the same manner and on the same grid.

\section{Calculation of Stress State}

Based on the obtained temperature distribution at half section 
cylinder $\quad T(r, z)(a \leq r \leq b ; 0 \leq z \leq H) \quad$ in accordance with the [14] are set dependences $\lambda(r, z)$, $\mu(r, z)$ and $K(r, z)$, that allows us to solve the boundary value problem for an inhomogeneous body, described by equations $[1,7]$

$$
\begin{aligned}
& \mu \nabla^{2} u+3(\lambda+\mu) \frac{\partial \varepsilon_{c p}}{\partial r}-\frac{\mu}{r^{2}} u+3 \frac{\partial \lambda}{\partial r} \varepsilon_{a v}+2 \frac{\partial \mu}{\partial r} \frac{\partial u}{\partial r}+ \\
& +\frac{\partial \mu}{\partial z}\left(\frac{\partial w}{\partial r}+\frac{\partial u}{\partial z}\right)-3 \frac{\partial}{\partial r}\left(K \varepsilon_{f}\right)=0 \\
& \mu \nabla^{2} w+3(\lambda+\mu) \frac{\partial \varepsilon_{c p}}{\partial z}+3 \frac{\partial \lambda}{\partial z} \varepsilon_{a v}+2 \frac{\partial \mu}{\partial z} \frac{\partial w}{\partial z}+ \\
& +\frac{\partial \mu}{\partial r}\left(\frac{\partial u}{\partial z}+\frac{\partial w}{\partial r}\right)-3 \frac{\partial}{\partial z}\left(K \varepsilon_{f}\right)=0
\end{aligned}
$$

with mixed boundary conditions (see. Fig. 1):

$$
\begin{aligned}
& z=0, \quad u=w=0 \\
& z=H, \quad \sigma_{z}=\tau_{r z}=0 \\
& r=(a, b), \quad \sigma_{r}=\tau_{r z}=0 . \\
& \operatorname{In}(5,6) \varepsilon_{a v}=\left(\varepsilon_{r}+\varepsilon_{\theta}+\varepsilon_{z}\right) / 3 \text {-average strain, }
\end{aligned}
$$

$\varepsilon_{f}$-forced (temperature) deformations.

Some results of calculations made by the variational-difference method shown in Fig. 4. Mechanical characteristics correspond to concrete and equal to $E=2 \cdot 10^{4} \mathrm{MPa} ; \quad v=0,2$; $\alpha_{T}=1 \cdot 10^{-5} 1 /{ }^{o} \mathrm{C}$. Provided graphs correspond to the homogeneous ( $\left.E=E_{0}, \lambda_{T}=\lambda_{T 0}\right)$ and inhomogeneous ( $\left.E=E(T), \lambda_{T}=\lambda_{T 0}\right)$ materials. The results of calculations obtained at $E=E(T), \lambda_{T}=\lambda_{T}(T)$, taking into account a increase the temperature in the last case, will correspond to curves lying between shown.
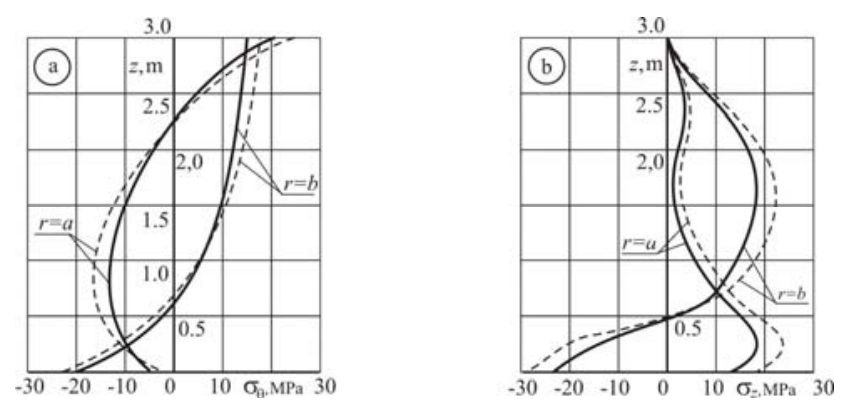

Fig. 4. Diagrams of thermal stresses $\sigma_{\theta}$ (a) and $\sigma_{z}$ (b) - - - - Homogeneous material; —_ Inhomogeneous material

From these results it follows that both (compressive and tensile) stresses reach a very significant absolute values comparable and higher than the design strength of the concrete. This should be reflected in the appointment of the scheme of reinforcement structure. Accounting the temperature inhomogeneity of the elastic modulus leads to a significant reduction (up to $20 \%$ ) in stresses that agrees qualitatively with the results of numerous calculations obtained earlier [6].

In [15] there was considered a similar problem of calculation the orthotropic cylinder with discrete changes in temperature on its inner surface. It was an analytical solution heat conduction problem in the Fourier series and the calculation of the stress state of the cylinder carried out variational-difference method. In the above-mentioned work was investigated the influence of two-dimensional temperature inhomogeneity of deformation properties of the material and orthotropy on the stress state related to the reinforcement of concrete cylinder.

\section{Conclusions}

From the presented results it is clear that the inhomogeneity of concrete at high temperatures leading to a decrease in the stiffness leads to reduction in stresses. This allows in design to reduce the thickness or decrease the reinforcement shell. Calculation of the shell located in a two-dimensional temperature field allows you to define areas tensile and compressive stresses. Averaging the temperature field provides only a rough estimate of the stress state.

\section{Acknowledgement}

Scientific work is realize under support of Ministry education and science Russian Federation (grant of President Russian Federation No14.Z57.14.6545-SS)

\section{References}

1. V.I. Andreev, Some problems and methods mechanics of nonhomogeneous bodies, Publ. house ASV, Moscow, 2002.

2. V.I. Andreev, Optimization of thick-walled shells based on solutions of inverse problems of the elastic theory for inhomogeneous bodies, Computer Aided Optimum Design in Engineering XII (OPTI XII). WIT Press. (2012) 189-201

3. V.I. Andreev, A.S. Chepurnenko, B.M.Yazyev, Model of Equal-stressed Cylinder based on the Mohr Failure Criterion. Advanced materials Research. 887-888 (2014) 869-872

4. V.I. Andreev, Stress-strain State of Weightless Inhomogeneous Array with Cylindrical Hole, Advanced Materials Research. 919-921 (2014) $740-743$ 
5. V.I. Andreev, Inverse problems of the inhomogeneous theory of elasticity for thick-walled shells, Journal "Computational Methods and Experimental Measurements", 2 No. 2 (2014) 202-216

6. V.I. Andreev, D.A. Kapliy, Stress state of a thick-walled cylindrical shell under the combined action of radiation and temperature field, Advanced Materials Research. 1006-1007 (2014) $177-180$

7. V.I. Andreev, Numerical-analytical solution of two-dimensional problem for elastic radially inhomogeneous thick-walled cylinder, Applied Mechanics and Materials. 752-753 (2015) 642-647

8. G.I. Zholdak, V.I. Ivanov, Construction dry protection nuclear reactor nuclear power plant // Problems of Atomic Science and Technology. Design and construction. 2 (1984) 35 - 40

9. N.P. Abovskiy, I.P. Andreev, A.P. Deruga, Variational principles of the theory of elasticity and the theory of shells, Nauka, Moscow,1978.
10. A.A. Samarskiy, V.B. Andreev, Difference methods for elliptic equations, Nauka, Moscow, 1976.

11. K. Vasidzu, Variational methods in the theory of elasticity and plasticity, Mir, Moscow, 1987.

12. B.K. Pergamenshchik, G.A. Lavdanskaya, Temperature field in a thick-walled cylinder with an internal heat source, Proceedings MISI. 56 (1968) $18-24$

13. V.B. Dubrovsky, Z. Oblevich, Building materials and constructions protection against ionizing radiation, Stroyizdat, Moscow, 1983

14. SNiP 2.03.04-84 Concrete and reinforced concrete structures, designed to work in conditions of elevated and high temperatures. CITP Gosstroy USSR, Moscow, 1985

15. V.I. Andreev, M.V. Belyj, S.P. Kirillov, Calculation of axisymmetric thermostressed state radially inhomogeneous cylinder, Building mechanics engineering designs, 6 (1996) 27-30 